\title{
Computer design color drawing
}

\begin{abstract}
A program is proposed for constructing multi-level color images of two-dimensional sections of any analytically defined functions $\mathrm{Z}=\mathrm{Z}(\mathrm{X}, \mathrm{Y})$ on the $\mathrm{X}, \mathrm{Y}$ plane at any scale on a computer display.

The program allows you to reflect on the screen in various colors located in the computer palette, any desired ranges of changes of the $\mathrm{Zi}$ function. The program can be used to automate or speed up the process of creating a geometric pattern of textile products (fabrics, knitwear, scarves, etc.). The work of the program is illustrated by the example of creating a variety of color patterns of carpets.
\end{abstract}

Keywords: automating the process of creating a color pattern, computer programs, textile materials
Volume 6 Issue 2 - 2020

\section{Pavel Shlyakhtenko}

Candidate of Physical and Mathematical Sciences, Doctor of Technical Sciences, Professor Emeritus, St. Petersburg State University of Industrial Technology and Design, Russia

Correspondence: Pavel Shlyakhtenko, Candidate of Physical and Mathematical Sciences, Doctor of Technical Sciences,

Professor Emeritus, St. Petersburg State University of Industrial Technology and Design, Russia, Email pavelsh12012@yandex.ru

Received: March 31, 2020 | Published: April 17, 2020

\section{Introduction}

Currently, a technology has been developed for the printer applying a color pattern to flat surfaces, as well as technology for automatically transferring a computer-generated image of a pattern to woven and knitted fabrics in the process of their controlled manufacture.

Unfortunately, the massive use of such technologies is hindered by the artist's low productivity. It is the unpredictable and significant duration of this initial part of the process, depending on the state of the artist's creative abilities at a particular moment, that is a natural brake on the path to the complete automation of the whole process, because the problems of automation of all subsequent stages of manufacturing industrial products for a specific drawing, in principle, already resolved.

There is a method of computer-aided construction of color drawings "according to fractals", that is, by special algorithms that support the principle of "likeness of small to large" or other conceptual mathematical principles ("Mandelbrot set", "Koch set"). In this method, drawing serves only as a graphic illustration of a mathematical concept. Moreover, it is not clear in advance what should result from the construction.

In a sense, working with "fractals" in relation to the applied problem of creating a variety of color patterns for textiles is similar to "finding a needle in a haystack", as evidenced by an extremely limited set of interesting patterns that are reproduced in each subsequent edition.

$\mathrm{In}^{2,3}$ a demo version of a sufficiently powerful program for constructing two-dimensional color images using the analytical formulas "EIDOSCOPE" is considered, illustrating the promising possibilities of its application. When working with the program, you recall a kaleido-osprey from distant childhood, thanks to which we could see an innumerable variety of fantastic color paintings, replacing each other at every turn. But the EIDOSCOPE program is immeasurably better, since it allows you to control and analyze experiments with various patterns of drawings, trying on them different color palettes. You can specify the formula of the picture, and the computer will recreate it on the screen, blooming in the colors you specified.
In memory of the developed program, which serves to demonstrate its capabilities, to date, more than 100 formulas have been laid down by which it can build practically countless variations of color images of drawings from 16 any (not necessarily different) colors. These colors can be taken in any random sequence from the colors stored in the computer's memory, or in a user-controlled mode, when one, two, three or four pictures from the regular (set) picture can be immediately displayed on the display, or random sequence.

Similarly, by choosing a picture, you can consider it in any of 70 different color palettes pre-selected in the program. All these operations can be observed in dynamics, when the drawings non-stop replaces each other on the screen. In this case, you can choose your favorite picture, stopping it, and then observe the dynamics of its decision in various color palettes. The program provides for almost unlimited possibilities for interfering with the parameters of a drawing (formula), its scale, color palette and color scheme, supplementing, refining and deleting elements of a picture and paint at its own discretion. The number of formulas entered into the program, the number of palettes, and the number of colors in each palette are, in principle, unlimited.

The possibilities of the discussed method of computer-aided construction of original drawings that are suitable, in the opinion of the author, for carpets are illustrated below.

\section{Results and discussion}

Figure 1 shows images of drawings constructed by a computer according to one formula for four successively applied color palettes (a) and two used palettes (b) and (c).

Figure 2 shows images of drawings constructed by a computer according to one formula for eight successively applied color palettes (a) and two used palettes (b) and (c).

Figure 3 shows an image of a drawing constructed by a computer according to the following formula in four color palettes (a) and a variant of the color scheme for a drawing using this formula (b).

Figure 4 presents figures constructed according to two new formulas and different color schemes of figure (a) and (b). 

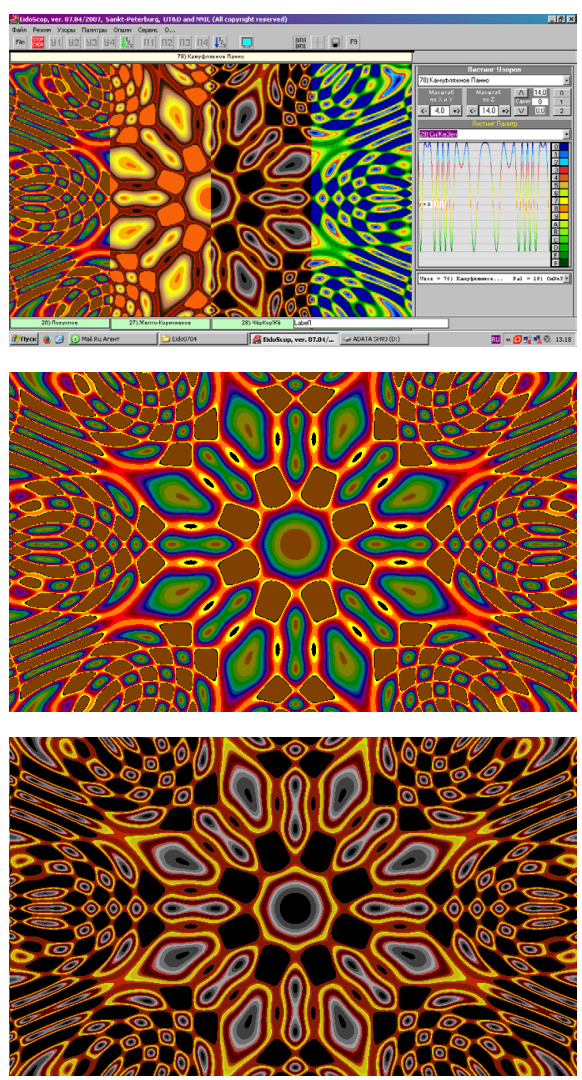

Figure I The construction of the figure according to the formula in four color palettes (a), the variants of the two color schemes of the figure according to this formula (b) and (c).
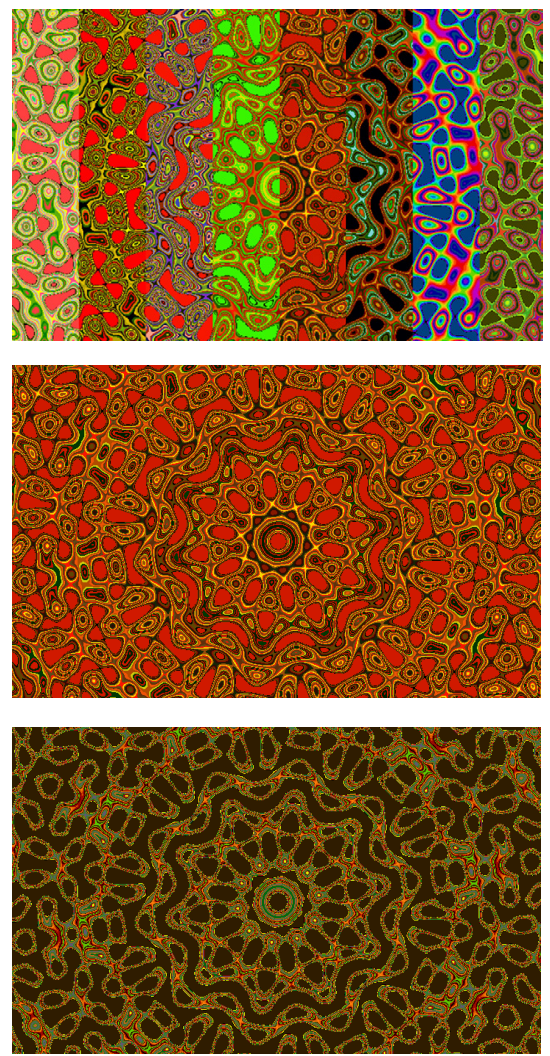

Figure 2 Drawing a pattern using a different formula in eight color palettes (a), options for two colors for a picture using this formula (b) and (c). 

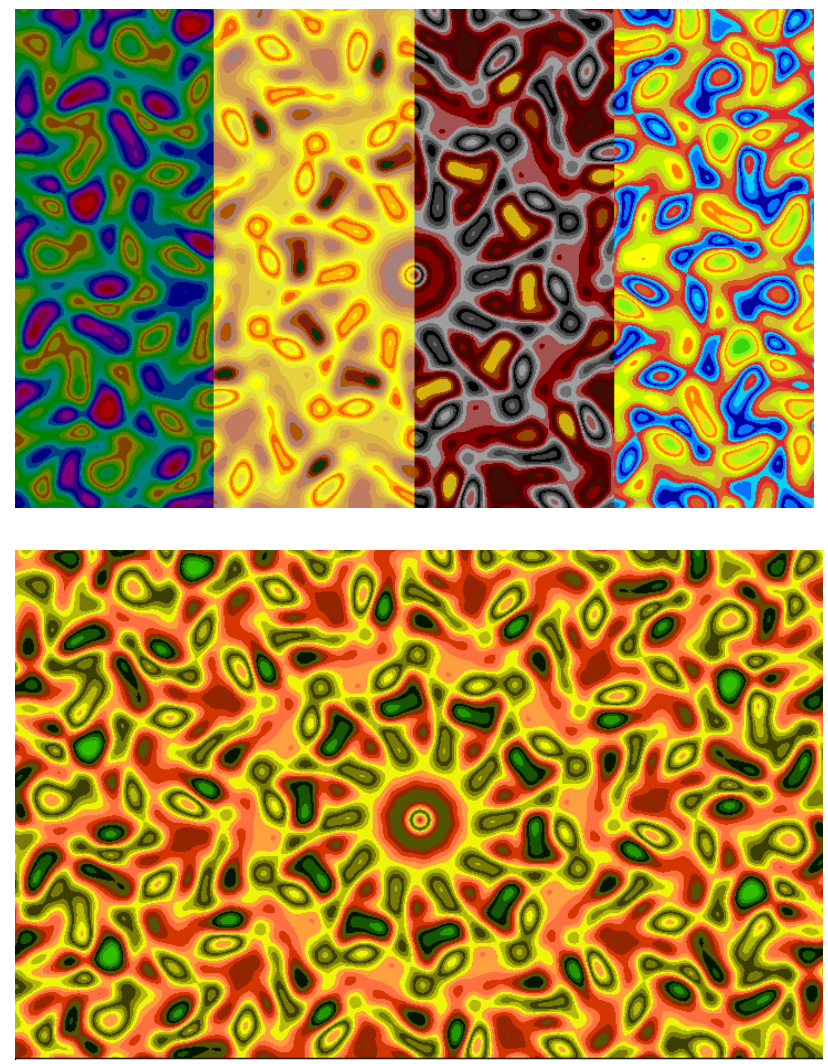

Figure 3 Building a picture using a different formula in four color palettes (a) and a color scheme for a picture using this formula (b).
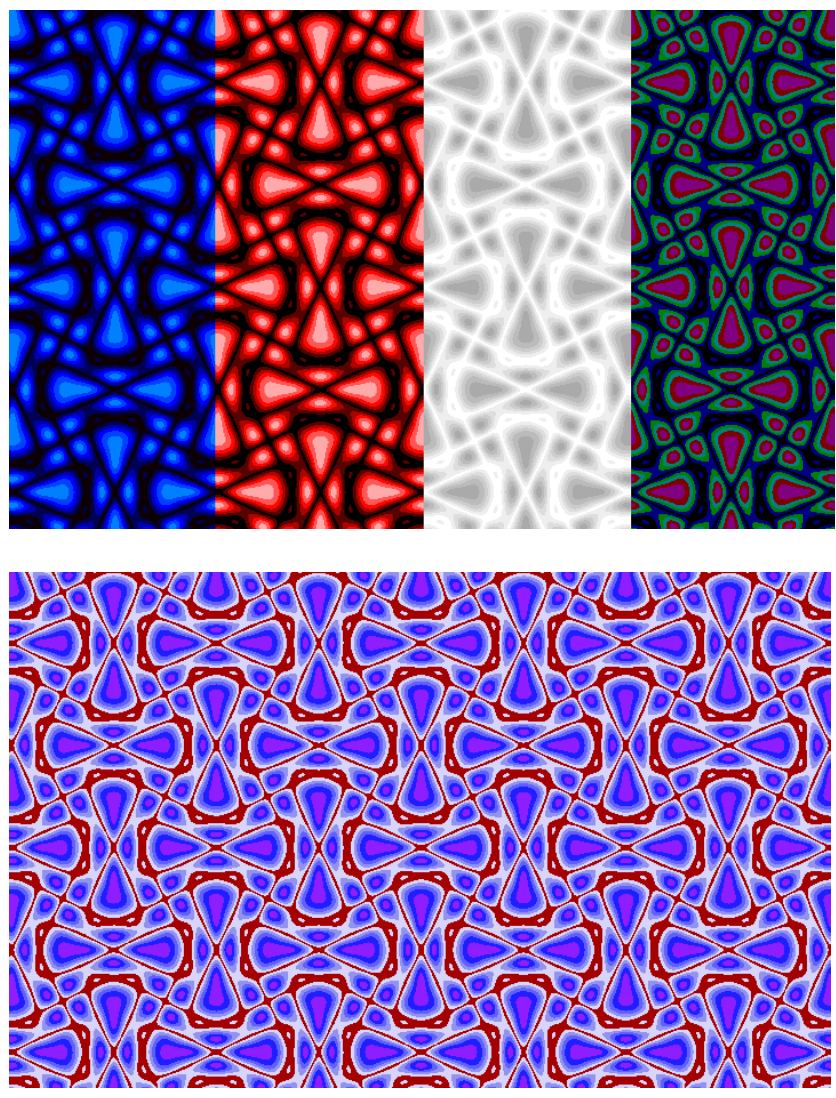

Figure 4 Drawings constructed using two new formulas and different color schemes of drawing (a) and (b). 
Figure 5 contains drawings constructed according to different formulas and differ-ent color schemes of figure (a) and (b).

Figure 6 presents the figures constructed by the computer according to one formula, a different size and different color schemes of figure (a) and (b). Figure 7 shows computer drawings constructed using the same formula and different palettes (a), (b) and (c).

From the analysis of long-term work with the program $c,^{3-5}$ taking into account the data presented in this paper, we can draw the following conclusions:

1. Formulas have been found that make it possible to assert that the computer-based color drawings built on them can be successfully used in various materials (carpets, scarves, tiles, mats, fabrics, bedding, linoleum, oilcloth).

2. The prospects and effectiveness of using the program for constructing countless variations of unique color patterns suitable for use in production are shown.

3. The program is promising when creating completely new technologies for storing and information about drawings in the advertising business, as it requires for storage and reproduction of drawings thousands of times less than the amount of computer memory.

4. The results of the work can be used to automate or speed up the procedure for creating a geometric pattern of textile products, that is, the most important, expensive and creative part of the technological process of building materials.

5. The program can be used to create fundamentally new technologies for storage, selection, production and sale of products using a unique, non-repeating pattern selected by the customer through the Internet for production. This paves the way for the creation of a new "breakthrough" technology for the production of material with a unique, non-repeating pattern via the Internet directly "to the customer", who in this case can itself be an accomplice in the process of creating the pattern.

6. The program can also be used as a textbook for students specializing in Computer Design, as well as in the form of a game that develops children's artistic taste and mathematical abilities.
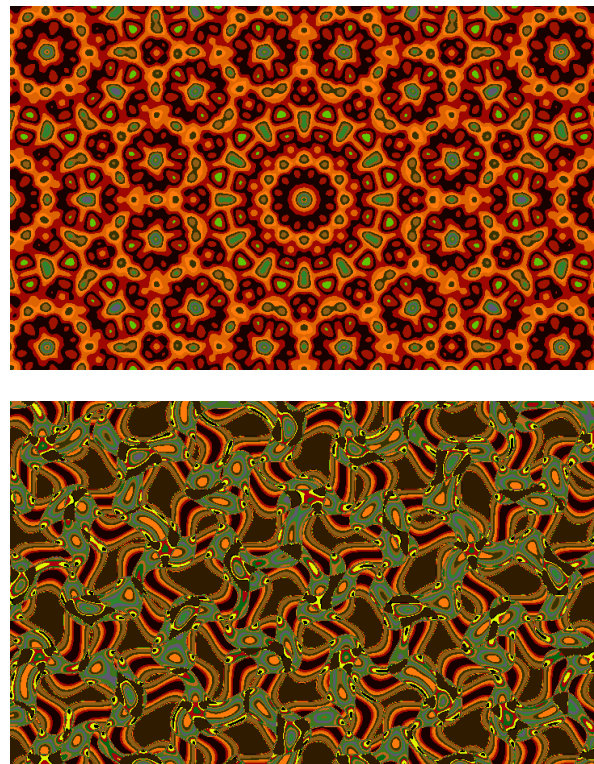

Figure 5 Drawings constructed by various formulas and different color schemes of drawing (a) and (b).
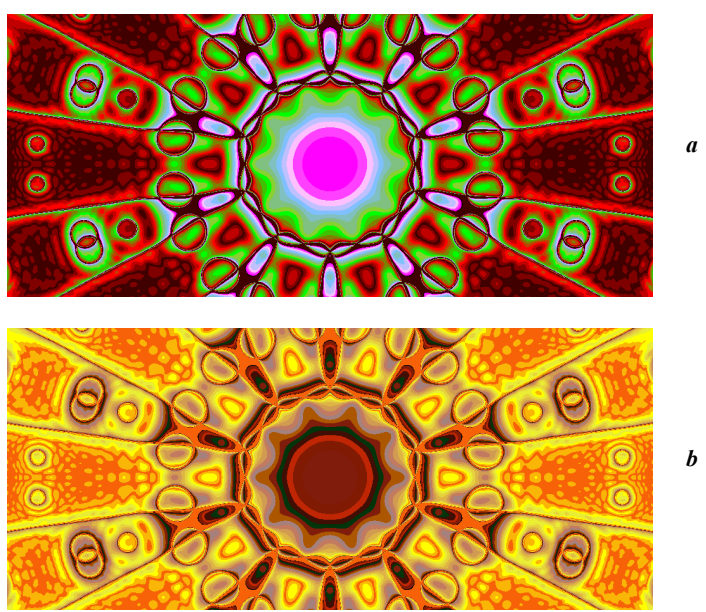

Figure 6 Drawings constructed according to one formula, different size and and different color schemes and drawing (a) and (b). 

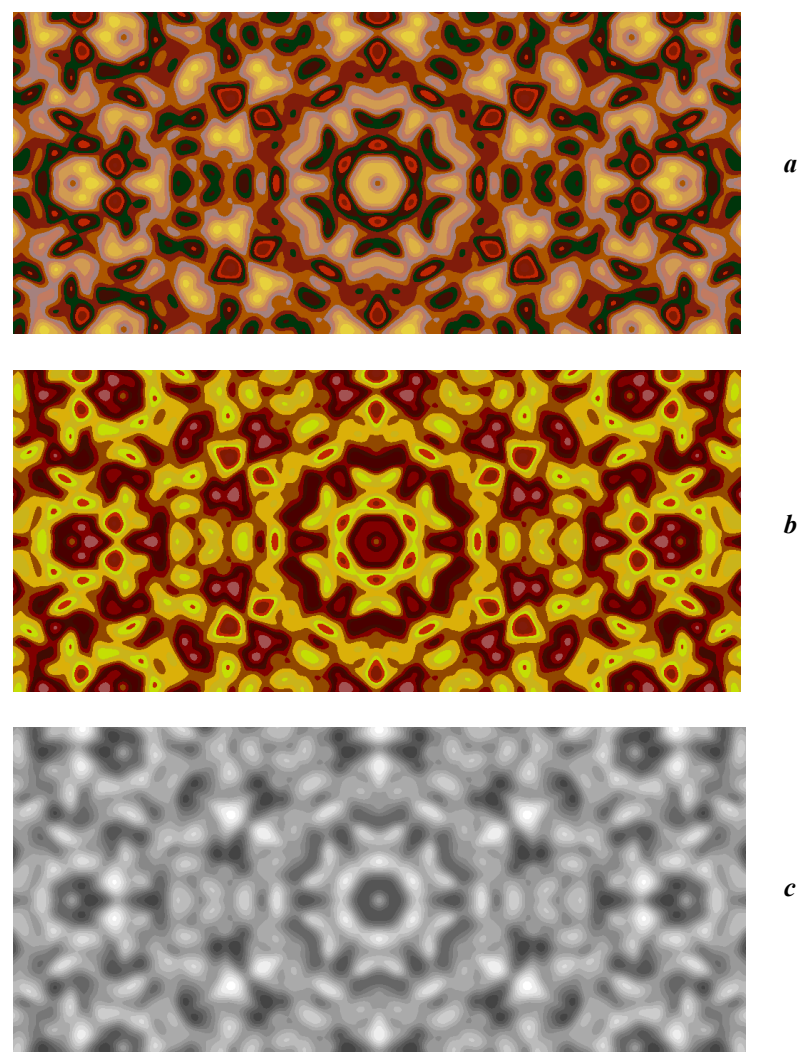

Figure 7 Drawings based on the same formula and different palettes (a), (b) and (c).

\section{Acknowledgments}

None.

\section{Funding}

None.

\section{Conflicts of interest}

Author declares there is no conflict of interest.

\section{References}

1. Paitgen XO, Rihter PX. Beauty fractals. 1993.
2. Shlyakhtenko PG, Nefedov VP. The program for the construction of numerous variants of wooden figures on analytical formulas. Zaregv Reestreprogrammdlia. Opubl Proguammidlia. 2007.

3. Shlyakhtenko PG. Non-destructive method of optical inspection of structural parameters of fiber materials. 2010.

4. Shlyakhtenko PG, Nefedov VP. Computer-aided color drawings with analytic formulas. Dizain Materiali Tehnologia. 2010;2:94-98.

5. Shlyakhtenko PG. Computer design of a variety of color drawings. COJ Technical \& Scientific Research. 2018;1(3):000514. 